\title{
Objective patient compliance in long-term use of nCPAP
}

\author{
B. Fleury*, D. Rakotonanahary**, C. Hausser-Hauw*, B. Lebeau*, C. Guilleminault+
}

Objective patient compliance in long-term use of $n C P A P$. B. Fleury, D. Rakotonanahary, C. Hausser-Hauw, B. Lebeau, C. Guilleminault. (C)ERS Journals Ltd 1996.

ABSTRACT: Concerns remain regarding patient compliance with nasal continuous positive airway pressure (nCPAP). Poor objective compliance during the first months of treatment has been reported, but no data are available among chronically treated patients.

Use of nCPAP, in 17 chronically treated obstructive sleep apnoea patients $(820 \pm 262$ days) was evaluated objectively using a pressure monitor (MC+; Sefam, France). Two consecutive recording periods of 30 sessions of treatment were scheduled at the patient's home. To minimize the potential bias caused by the introduction of the monitor, only the pressure data obtained at the end of the second period of recording (T2) were analysed.

During the $28.1 \pm 2.6$ monitored days, the mean effective daily rate of use was $7.1 \pm 1.1 \mathrm{~h}, 97 \%$ of the rate indicated by the standard in-built time counter. The prescribed pressure was observed during $95 \%$ of the machine run time. The nCPAP system was used for $94 \%$ of the monitored days. Sixty percent of the patients used their device every day.

These preliminary results suggest that, contrary to reported compliance during the early period of the treatment, objective use of nasal continuous positive airway pressure therapy in chronically treated patients is satisfactory. Eur Respir J., 1996, 9, 2356-2359.
*Unité de Sommeil, Service de Pneumologie, Hôpital Saint Antoine, Paris, France. **Centre de Diagnostic et de Traitement des Affections Respiratoires, Paris, France. +Stanford University, Sleep Disorders Center, Palo Alto, California, USA.

Correspondence: B. Fleury

Unité de Sommeil

Service de Pneumologie

Hôpital Saint Antoine

184 rue du Faubourg Saint Antoine Paris 75012

France

Keywords: Chronic daily use compliance

nasal continuous positive airway pressure obstructive sleep apnoea syndrome sleep apnoea

surveillance of nasal continuous positive airway pressure use

Received: December 281995

Accepted after revision July 51996
Although nasal continuous positive airway pressure (nCPAP) has so far been the most effective treatment available for obstructive sleep apnoea syndrome (OSAS) [1], concerns remain regarding patient compliance. Until recently, objective evaluation of compliance was based solely on time counters built into the nCPAP device. These counters measure the cumulative time that the apparatus is turned on [2-5], but provide no information on the schedule and amount of time the device is used each day. The long-term recording of the pressure in the nCPAP equipment has recently provided a more precise index of equipment use by measuring time spent with the facial mask on [6-8]. These studies found that compliance with nCPAP was generally poor during the first months of treatment; however, they offered no data on chronically treated patients. The individual (residual daytime somnolence and cardiovascular morbidity) and economic cost of the collective misuse of nCPAP therapy would be significant. In France, more than 10,000 OSAS patients are chronically treated at home, representing an annual expense of approximately 20,000,000 \$US for the health insurance system. The goal of the present study was to perform an objective evaluation of compliance, using a pressure monitor, in a group of OSAS patients treated with nCPAP for at least 1 year.

\section{Methods}

\section{Subjects}

Forty nine patients were identified, who had been diagnosed with OSAS after two nights of polygraphic monitoring at the Centre de Traitement des Affections Respiratoires (Paris, France) and had been prescribed nCPAP for more than a year.

Most nCPAP devices have the same efficacy and the selection of equipment to be used for treatment of OSAS patients is left to the medical device providers, in this case C.A.R.D.I.F. (Ivry, France). This allocation is performed independently of any clinical parameters. Thus, these patients were supplied with equipment from different manufacturers that included: the Sleep Easy III ${ }^{\mathrm{TM}}$; the REM Star ${ }^{\mathrm{TM}}$; the Morphée ${ }^{\mathrm{TM}}$; and the Sefam REM+ ${ }^{\mathrm{TM}}$, or Sefam REM+ control ${ }^{\mathrm{TM}}$.

The prospective investigation was possible due to the availability of a microprocessor (MC+; Sefam, France) that allows monitoring of pressure in the pneumatic circuitry of the nCPAP equipment. This microprocessor is only compatible with equipment from the same company (Sefam. Inc.) and only 17 of the 49 subjects used equipment manufactured by Sefam. These 17 subjects (3 females and 14 males) represent the study group. They had been prescribed nCPAP and had been followed by the patient care association for $820 \pm 262$ days. Their mean age was $61 \pm 11 \mathrm{yrs}$, their mean body mass index (BMI) was $30.5 \pm 4.6 \mathrm{~kg} \cdot \mathrm{m}^{-2}$ and their mean nCPAP level was $9.5 \pm 1.6 \mathrm{cmH}_{2} \mathrm{O}$. This subgroup of 17 patients did not differ statistically from the total initial group in terms of age, BMI, initial polysomnographic findings, and daily rate of use of the nCPAP equipment as indicated by a built-in time counter or duration of follow-up (table 1). All 17 subjects had given written informed consent to participate in a study of long-term effects of nCPAP use approved by the Institutional Internal Review Board. 
Table 1. - Anthropometric and polysomnographic characteristics of the 17 evaluable patients and of the 49 initial patients

\begin{tabular}{|c|c|c|c|c|}
\hline & $\begin{array}{c}\text { Evaluable } \\
\text { patients } \\
n=17\end{array}$ & $\begin{array}{c}\text { Initial } \\
\text { patients } \\
\mathrm{n}=49\end{array}$ & F-value & $\mathrm{p}$-value \\
\hline BMI $\mathrm{kg} \cdot \mathrm{m}^{-2}$ & $30.5 \pm 4.6$ & $31.8 \pm 6.3$ & 1.66 & 0.12 \\
\hline Age yrs & $61 \pm 11$ & $58 \pm 10$ & 0.89 & 0.11 \\
\hline $\operatorname{Sex} \quad M / F$ & $14 / 3$ & $41 / 8$ & 0.91 & 0.56 \\
\hline TST min & $383 \pm 48$ & $371 \pm 54$ & 1.37 & 0.25 \\
\hline Stage $1 \%$ TST & $49 \pm 15$ & $47 \pm 15$ & 1.05 & 0.49 \\
\hline Stage $2 \%$ TST & $34 \pm 10$ & $37 \pm 12$ & 1.45 & 0.21 \\
\hline Stage $3 \%$ TST & $9.5 \pm 9.3$ & $9.6 \pm 8.2$ & 0.82 & 0.66 \\
\hline Stage $4 \%$ TST & $1.2 \pm 2.5$ & $0.8 \pm 1.9$ & 0.73 & 0.75 \\
\hline REM sleep \%TST & $6.2 \pm 4.3$ & $6.2 \pm 4.3$ & 0.97 & 0.49 \\
\hline AHI events $\cdot h^{-1}$ & $44.3 \pm 21.2$ & $50.9 \pm 25.3$ & 1.31 & 0.27 \\
\hline Mean $\mathrm{Sa}_{\mathrm{a}, \mathrm{O}_{2}} \%$ & $92 \pm 3$ & $92 \pm 3$ & 1.01 & 0.53 \\
\hline $\operatorname{Min} \mathrm{S}_{\mathrm{a}, \mathrm{O}_{2}} \%$ & $65 \pm 12$ & $69 \pm 12$ & 1.08 & 0.46 \\
\hline nCPAP $\mathrm{cmH}_{2} \mathrm{O}$ & $9.5 \pm 1.6$ & $10.1 \pm 1.7$ & 1.15 & 0.39 \\
\hline Follow-up days & $820 \pm 263$ & $772 \pm 267$ & 1.01 & 0.51 \\
\hline Daily rate of use at $T 0 h$ & $6.8 \pm 1.2$ & $6.7 \pm 1.3$ & 1.01 & 0.52 \\
\hline
\end{tabular}

Values are presented as mean \pm SD. Comparisons were by Fisher's test. BMI: body mass index; F: female; M: male; TST: total sleep time; REM: rapid eye movement; AHI: apnoea hypopnoea index; $S_{\mathrm{a}, \mathrm{O}_{2}}$ : arterial oxyhaemoglobin saturation; Min: minimal; T0: day of entry into the study; nCPAP: nasal continuous positive airway pressure.

\section{MC+ monitor}

The $\mathrm{MC}+$ monitor is equipped with an Intel 8051 microprocessor, $16 \mathrm{~K}$ of nonvolatile memory (SRAM) chip, an 8 bit analogue-to-digital (A/D) converter, a battery back-up, a real-time clock, and a power-on reset circuit. The variable component of the pressure signal given by the pressure transducer (Sensym SCX01) is analysed in order to determine whether the patient is breathing into the mask. Events are detected as "power on"/"power off", and "mask on"/"mask off". Up to 30 consecutive "sessions"of treatment can be recorded before having to download the information. One "session" is the time from "power on" to "power off".

\section{Procedure}

All 17 patients were included in the study, which was performed during two consecutive months. Subjects were told that the $\mathrm{MC}+$ was installed to ensure that the nCPAP equipment was functioning properly, but were not informed about the equipment's ability to monitor their daily use of the nCPAP device. The MC+ was installed in the continuous positive airway pressure (CPAP) circuitry at the patient's home by a staff physician from the sleep laboratory of the Service de Pneumologie, Hôpital Saint Antoine (Paris, France). Two consecutive periods of thirty sessions each were monitored.

\section{Data Analysis}

Information from the in-built counter. The mean daily rate of use determined from the in-built time counter (TC) was calculated by dividing the difference between two successive readings by the number of days elapsed. The data were obtained at the time of entry into the study (TC0), and at the end of each of the two consecutive study periods, called $\mathrm{TC} 1$ and $\mathrm{TC}_{2}$.
Information from the MC+ monitor. The mean effective daily rate of use (EU) was derived from the MC+ monitor readings. It was calculated by dividing the cumulative time with the mask on by the number of days the machine was actually used. The use of the machine at the prescribed nasal positive pressure was calculated as a percentage by dividing the cumulative time with the mask on by the cumulative time the machine was turned on. The percentage of the monitored day during which the machine was turned on and during which it was effectively used was also determined. To minimize any potential bias related to the installation of the $\mathrm{MC}+$ monitor on the nCPAP use, statistical analyses were performed only on the data from the second monitoring period (i.e. $\mathrm{T} 2, \mathrm{TC} 2$, and $\mathrm{EU} 2$ ).

\section{Statistical analyses}

For group data, the results are expressed as mean \pm SD. Fisher's test and unpaired t-test were used to compare unpaired groups. A paired t-test was carried out to determine differences between indices of nCPAP use, and the statistical significance was set at a p-value of less than 0.05 .

\section{Results}

Fourteen out of 17 patients reported side-effects with nCPAP. A humidifier had been added in 12 cases (six nasal dryness and six rhinitis), and the mask had been changed in two patients due to skin lesions of the nasal bridge. At entry to the study (T0), complaints of nasal dryness in three cases and rhinitis in four were still present despite the use of the humidifier and medical treatment. The patients, however, did not consider these side-effects sufficiently bothersome to limit their compliance with nCPAP therapy.

After the first 30 session recording period $(24.7 \pm 4.5$ days), two of the 17 subjects refused to participate 
in the second recording period, due to an unplanned journey in one case and the report of "discomfort" caused by the addition of the MC+ monitor in the other. Both subjects were regular nCPAP users according both to the time counter at T0 (5.2 and $6.5 \mathrm{~h}$ daily) and the pressure monitor readings at $\mathrm{T} 1$ (6.6 and $5.7 \mathrm{~h}$ daily).

The remaining 15 patients were recorded for $28.1 \pm 2.5$ additional days (median of 28 days, range 24-34 days). At T2, the mean daily rate of use calculated from the time counter ( $\mathrm{TC}_{2}$ ) was not different from the mean rate of use during the $247 \pm 45$ days preceding entry into the study (TC0), 7.1 \pm 1.1 vs $6.9 \pm 1.2 \mathrm{~h}$ (NS). The mean effective daily rate of use (EU2) was $6.9 \pm 1.3 \mathrm{~h}$. The individual rates of use are presented in figure 1 . Use of nCPAP, at the prescribed pressure, was observed for $95 \pm 14 \%$ of the machine run time (median $99 \%$, range $43-100 \%$, lower quartile $98 \%$ ). The least compliant patient was hospitalized during the recording period and there was a clear discrepancy between the mean rate of use from the time counter $(7.8 \mathrm{~h})$ and the pressure monitor $(1.8 \mathrm{~h})$ in his case. Two patients turned the equipment on several times a day (related to daytime napping and awakenings during the night with interruption of nCPAP use), exceeding the memory capacity of the $\mathrm{MC}+$ during the monitored period. These two subjects used their nCPAP equipment every day during 24 and 28 consecutive days at the prescribed pressures during 99 and $100 \%$ of the machine run time. The remaining 13 patients used their nCPAP system during $26.4 \pm 2.3$ days (median 27 days, range 22-30 days). This represents $94 \%$ of the monitored period (median 100\%, range 79-100\%).

Seven of the 13 patients $(54 \%)$ used their nCPAP device every day during the monitored period. In these seven patients, TC2 $(7.3 \pm 1.3 \mathrm{~h})$ was similar to EU2 $(7.3 \pm 1.3$ h). The last six subjects had more irregular compliance (79-97\% of the monitored period). Two of these more intermittent users were hospitalized, for 1 and 5 days, respectively, and did not use their nCPAP machines in the hospital. The four other less compliant users stopped their treatment for 1 to 7 days $(3,7,18$ and $21 \%$ of the

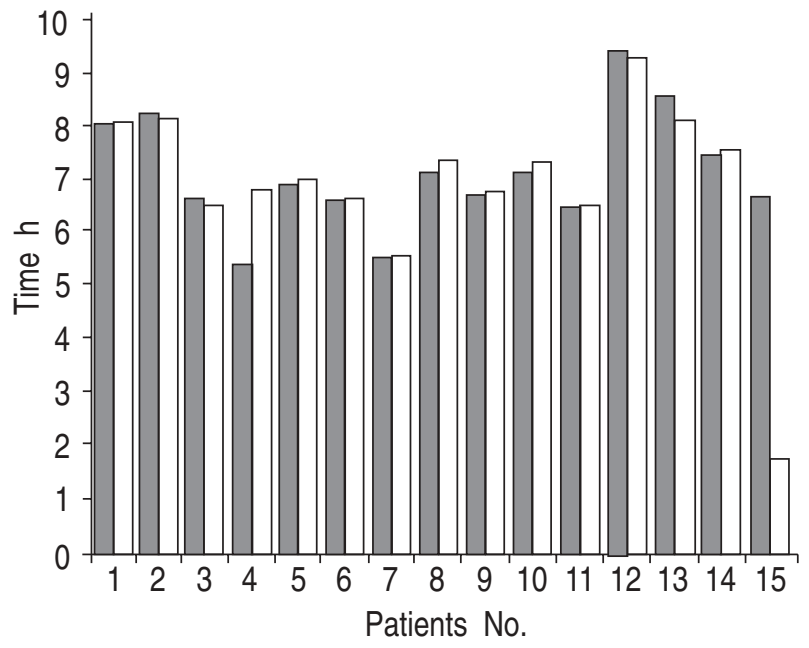

Fig. 1. - Individual rates of nasal continuous positive airway pressure (nCPAP) use. TC2: mean daily rate of use from the built-in time counter at the end of the 2 nd period of recording; EU2: mean effective daily rate of use from the pressure monitor at the end of the 2nd period of recording. $\square$ : $\mathrm{TC}_{2} ; \square$ : EU2. monitored period). A posteriori interviews revealed that these subjects were travelling at the time of noncompliance, but they used their system on all of the remaining nights, while at home.

\section{Discussion}

The MC+ monitor was selected because it had the advantage of being an accurate and well-tested monitor, easy to install in an existing nCPAP machine, and specifically designed to monitor the machine's pressure levels and time of delivery accurately. Finally, the fact that it was from the same manufacturer as the nCPAP equipment reinforced, to some extent, the explanation provided to the patients, i.e. a device to evaluate whether the machine was working properly. We acknowledge, however, that the selection of this monitor effectively reduced the population available for the study, as only 17 patients had received a compatible nCPAP. As shown in table 1, these subjects did not differ statistically from the total group. One may argue that the nCPAP in this investigation was more comfortable than those of other companies, but clinical experience indicates that this is not the case, as does the reported number of clinical problems associated with CPAP use.

The few studies performed to objectively assess compliance, using a pressure transducer incorporated into the pneumatic circuitry of the CPAP machine, describe poor use of the equipment $[6,7]$. In a bi-centric study by KRIBBS et al. [6], less than half of the 35 studied subjects used their nCPAP for at least $4 \mathrm{~h}$ daily, and only $5.7 \%$ used it at least $7 \mathrm{~h}$ on $70 \%$ of the monitored days. The authors concluded that actual nCPAP use fell short of the therapeutic goal of providing quality sleep all night, every night. However, this study was performed, primarily during the first 3 months of treatment, and a fraction of the new patients, most likely the least compliant, stopped their treatment after a few weeks, possibly making the rest of the group appear less compliant [7]. More recently, ReEves-Hoché and co-workers [7] reported an effective use of approximately $4 \mathrm{~h}$ each night and a compliance rate (machine run time/reported hours of sleep) of $68 \%$ in patients who continued nCPAP use for 6 months. In our subjects who had used CPAP for at least one year, effective use of the nCPAP was $94 \%$ of the monitored period, with $60 \%$ using the machine every day. The results show a better objective compliance to therapy in this group than those of the two previous studies. Hence, the length of time the CPAP has been used could strongly influence the results of an analysis of compliance.

Our study provides information on a different group of subjects: those who did not reject the treatment early on and were chronically treated. Recently, PIETERs et al. [9] reported the data on their first 95 patients with confirmed OSAS in whom compliance data were acquired for at least one year. The study showed that compliance to nCPAP treatment was reasonably good during a relatively long follow-up period of more than 2 yrs on average. Seventy four percent of patients used their CPAP device for more than $4 \mathrm{~h} \cdot$ day $^{-1}$, with a median compliance calculated from the built-in time counter of $5.2 \mathrm{~h}$. As such, our objective measurements of the compliance complement these recently published European data. 
Interestingly, we found that the mean effective daily rate of use corresponded to $97 \%$ of the rate of use indicated by the built-in time counter of the machine. This is an important point, although "spy" chips and monitoring devices are still uncommon, counters are present in many CPAP machines. In a previous study, we reported an averaged compliance rate, estimated from the CPAP's time counter, of $6.7 \mathrm{~h}$ each night among the patients followed [4]. It was of the same order of magnitude as the rate reported by KRIEGER [3] in a French population. The compliance of the patients at entry to the present study, $6.9 \mathrm{~h}$, was close to these values and it argues for the representativeness of the population studied.

Here, instead of presenting "compliance" data, we have reported our data in hours of effective use. This was done due to the lack of a standard definition of "compliance" of nCPAP use in the literature. This absence of definition made it difficult to make comparisons with the results of the previous studies. In addition, we did not want to influence the patients' behaviour by asking them to maintain a sleep diary, particularly at the time of the monitoring sessions. This would possibly, have, changed patient habits and undoubtedly drawn attention toward an investigation of nocturnal sleep. Although there was a possibility in our study that the introduction of the MC+ monitor into the patient's home could induce a modification of the patient's behaviour, our results suggest that not much modification occurred, as time counter values of mean daily use were not different at $\mathrm{T} 0$ and $\mathrm{T} 2$. The effective rate of use of the nCPAP system was satisfactory and it was noticeable that the rates of use estimated from the built-in time counter and from the pressure monitor were not significantly different. Lower uses (79 and $82 \%$ of the monitored period) were observed during hospitalization of otherwise regular users.

Our investigation indicates that, when hospitalized, patients do not appear to receive their prescribed treatment (i.e. nCPAP), or at least do not use the equipment appropriately, as demonstrated by the two subjects who, though compliant when at home, did not use their nCPAP machines in the hospital. This finding should remind practitioners that: 1) patients may not bring their equipment to a hospital, supposedly full of medical devices, if not told to do so; and 2) medical and nursing staff may need to be reminded that prescribed medical devices for use during sleep may be integral to the outcome of any intercurrent illness. At times, hospital staff may rely entirely on the written orders of the hospital's attending physician and forget, if not reminded, about chronic home care. Finally, our results support clinical wisdom, i.e. often patients do not like to carry their CPAP while travelling. This should encourage manufacturers to build equipment that is compact and that adapts to different voltages.

Finally, one may question why we had what could be viewed as excellent use in our population. Our patients had the opportunity to choose surgical treatment, as an alternative to nCPAP, at the initiation of the therapy and at each of the regular follow-up visits. After 1 year of regular treatment, they had experienced the disadvantages and advantages and preferred to keep the nCPAP therapy. All of them reported a dramatic improvement in their daily life with treatment. Most of them had tried a temporary cessation of treatment and had experienced a return of daytime somnolence shortly thereafter. The benefits of regular nasal continuous positive airway pressure use probably explain much of the good compliance observed in this group of chronically treated patients.

\section{References}

1. Sullivan CE, Berthon Jones M, Issa F, Eves L. Reversal of obstructive sleep apnoea by continuous positive airway pressure through the nares. Lancet 1981; i: 862-865.

2. Krieger J, Kurtz D. Objective measurement of compliance with nasal CPAP treatment for obstructive sleep apnoea syndrome. Eur Respir J 1988; 1: 436-438.

3. Krieger J. Long-term compliance with nasal continuous positive airway pressure (CPAP) in obstructive sleep apnea patients and nonapneic snorers. Sleep 1992; 15: S42-S46.

4. Fleury B, Rakotonanahary D, Tehindrazanarivelo AD, Hausser-Hauw C, Lebeau B. Long-term compliance to continuous positive airway pressure therapy (nCPAP) set up during a split night polysomnography. Sleep 1994; 17: 512-515.

5. Meurice JC, Dore P, Paquereau J, et al. Predictive factors of long-term compliance with nasal continuous positive airway pressure treatment in sleep apnea syndrome. Chest 1994; 105: 429-433.

6. Kribbs BN, Pack AI, Kline LR, et al. Objective measurement of patterns of nasal CPAP use by patients with obstructive sleep apnea. Am Rev Respir Dis 1993; 147: 887-895.

7. Reeves-Hoché MK, Meck R, Zwillich CW. Nasal CPAP: an objective evaluation of patient compliance. Am J Respir Crit Care Med 1994; 149: 149-154.

8. Reeves-Hoché MK, Hudgel DW, Meck R, Witteman R, Ross A, Zwillich CW. Continuous versus bi-level positive airway pressure for obstructive sleep apnea. Am J Respir Crit Care Med 1995; 151: 443-449.

9. Pieters Th, Collard Ph, Aubert G, Dury M, Delguste P, Rodenstein DO. Acceptance and long-term compliance with nCPAP in patients with obstructive sleep apnoea syndrome. Eur Respir J 1996; 9: 939-944. 